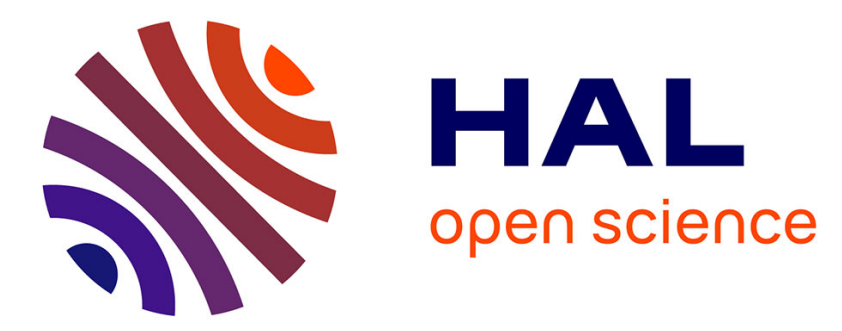

\title{
Organisation de la formation initiale des étudiants en pharmacie
}

Élisabeth Adenot, Laetitia Agay, Jean-Paul Belon, Arthur Piraux

\section{To cite this version:}

Élisabeth Adenot, Laetitia Agay, Jean-Paul Belon, Arthur Piraux. Organisation de la formation initiale des étudiants en pharmacie. Actualités Pharmaceutiques, 2019, 58, pp.25 - 28. 10.1016/j.actpha.2018.12.006 . hal-03486091

\section{HAL Id: hal-03486091 \\ https://hal.science/hal-03486091}

Submitted on 20 Dec 2021

HAL is a multi-disciplinary open access archive for the deposit and dissemination of scientific research documents, whether they are published or not. The documents may come from teaching and research institutions in France or abroad, or from public or private research centers.
L'archive ouverte pluridisciplinaire HAL, est destinée au dépôt et à la diffusion de documents scientifiques de niveau recherche, publiés ou non, émanant des établissements d'enseignement et de recherche français ou étrangers, des laboratoires publics ou privés.

\section{다)(1) $(5$}

Distributed under a Creative Commons Attribution - NonCommerciall 4.0 International 
Version of Record: https://www.sciencedirect.com/science/article/pii/S0515370018304877

Manuscript_19f677813411f0f35007dccc826764bd

Dochead dossier

Sous-dochead Les nouveaux enjeux de la formation du pharmacien

\title{
Organisation de la formation initiale des étudiants en pharmacie
}

\author{
Élisabeth Adenot ${ }^{\mathrm{a}, *}$, \\ Étudiante en troisième année des études pharmaceutiques, secrétaire générale et attachée \\ de presse de l'Anepf \\ Laetitia Agay ${ }^{c}$ \\ Pharmacienne, vice-présidente en charge de la communication à l'Anepf 2016-2017 \\ Jean-Paul Belon $^{d}$ \\ Professeur des Universités, ancien membre de la CPNEP et de la CPNES

\section{Arthur Piraux ${ }^{\mathrm{e}}$}

Pharmacien, vice-président en charge de l'enseignement supérieur à l'Anepf 2016-2017

aUniversité Paris-Descartes, 4 avenue de l'Observatoire, 75006 Paris, France

${ }^{b}$ Anepf, 4 avenue Ruysdaël, 75008 Paris, France

${ }^{`} 6$ chemin du Hameau-Blanc, 38240 Meylan, France

dUFR des sciences de santé, Université de Bourgogne, 7 boulevard Jeanne-d'Arc, 21000 Dijon, France

e 68 chemin des Bordages, 72230 Arnage, France

*Auteur correspondant.

Adresse e-mail : presse@anepf.org (É. Adenot).

Résumé

C'est durant leurs études que les étudiants en pharmacie apprennent toutes les bases de leur futur métier. Officine, internat, industrie, recherche, le champ des possibles est vaste et permet d'explorer 
de nombreuses voies, qu'elles soient au contact du patient ou dédiées à la préparation de ses médicaments et à la surveillance de ces derniers.

(c) 2018

Mots clés - études ; industrie ; internat ; officine ; recherche ; université ; stage

Summary à venir

(c) 2018

Keywords à venir

L'organisation actuelle des études de pharmacie est issue d'un long processus de réformes successives. La formation se décompose en trois niveaux (figure 1) et fait la part belle aux stages (encadré 1).

Encadré 1. Les stages, de la théorie à la pratique

Les stages occupent une place importante au sein du cursus d'études, raison pour laquelle la formation est qualifiée de professionnalisante. Différentes typologies de stages, avec des objectifs précis et des pratiques pédagogiques spécifiques, permettent à l'étudiant de parfaire son projet professionnel et de mettre en pratique ses connaissances.

TEG1 Le stage d'initiation de deuxième année se définit comme un stage d'observation durant lequel l'étudiant découvre le métier premier de pharmacien : celui de pharmacien d'officine. D'une durée minimale de six semaines, il permet d'appréhender le fonctionnement d'une officine ainsi que les rapports que peut entretenir le pharmacien avec les patients et les autres professionnels de santé.

TEG1 Les stages d'application de troisième et de quatrième année durent une à deux semaines selon les facultés. L'étudiant doit y met en pratique ses connaissances théoriques concernant une thématique précise, à partir de situations issues du terrain.

TEG1 La cinquième année dite "hospitalo-universitaire" octroie à l'étudiant le statut d'étudianthospitalier (externe) lui permettant de toucher une rémunération (de l'ordre de $200 €$ par mois pendant douze mois si le stage est réalisé à temps partiel). Le stage peut être effectué dans un service clinique, en PUI, laboratoire de biologie, dans un centre hospitalier étranger, etc. Il s'agit, pour l'étudiant, de trouver sa place, et de facto celle du pharmacien, dans le milieu hospitalier en collaboration avec les autres professionnels de santé. Il doit également réaliser des actions de prévention sur le terrain, correspondant à la phase d'action du service sanitaire.

TEG1 Enfin, le stage de sixième année destiné aux officinaux repose sur de la pratique pure, permettant une transition entre les bancs de la faculté et le milieu professionnel. Puisqu'il dure six mois, l'étudiant a le temps de s'acclimater à une officine, donc à une équipe et une patientèle, mais 
aussi de mettre en place différentes actions (prévention, qualité à l'officine, entretiens, etc.). Une gratification lui est accordée (536,80 € par mois). Dès la rentrée 2019, ce stage se décomposera différemment : trois mois à l'officine, puis trois mois de cours et, enfin, six mois dans un établissement libre. Un changement du statut de l'étudiant est à prévoir. La gratification sera alors réévaluée.

T1 La première année commune aux études de santé

TEG1 Depuis 2010, la première année commune aux études de santé (Paces) a remplacé la première année du premier cycle des études de pharmacie (PCEP 1). Elle regroupe les étudiants se destinant aux études de pharmacie, médecine, maïeutique et odontologie mais aussi, selon les universités, aux études de kinésithérapie, ergothérapie et de psychomotricien. Pour chaque formation et université, un numerus clausus est déterminé annuellement [1]. À l'issue du concours qui clôt cette première année, les étudiants sont classés en fonction de leurs résultats dans chacune des filières.

TEG1 Pour faire face à la difficulté de la Paces, un grand nombre d'étudiants font appel à leurs pairs (des étudiants de deuxième ou troisième année des formations de santé) à travers le tutorat. Des exercices, des concours blancs, des cours de méthodologie, etc., leur sont proposés.

TEG1 Depuis 2013, et devant l'échec relatif de la Paces par rapport aux objectifs initiaux, la loi autorise des expérimentations concernant les modalités d'admission des étudiants dans les études de santé [2]. Les premières ont débuté en 2014 et 2015 dans dix universités. Une seconde vague a été autorisée par le ministère à la rentrée 2017 ou en 2018 dans huit autres universités.

TEG1 En septembre 2018, le président de la République a annoncé, dans le Plan Santé 2022, une réforme du système d'entrée dans les études de santé, mettant fin au numerus clausus et à la Paces [3].

\section{L’Association nationale des étudiants en pharmacie de France (Anepf) aux côtés des étudiants durant leurs études}

L'Association nationale des étudiants en pharmacie de France (Anepf) ${ }^{1}$ est une association à but non lucratif fondée le 14 mars 1968 à Paris. Elle regroupe les 24 associations étudiantes des 24 facultés de pharmacie du territoire et représente ainsi les 33000 étudiants en pharmacie de l'Hexagone. Elle s'est dotée également de différents réseaux - santé publique, humanitaire, international, tutorat et industrie -, qui témoignent de la grande implication de la génération des pharmaciens de demain dans les engagements solidaires, l'accompagnement des pairs ou le rayonnement de la profession.

${ }^{1}$ www.anepf.org 


\section{T1 Le diplôme de formation générale en sciences pharmaceutiques}

TEG1 Le diplôme de formation générale en sciences pharmaceutiques (DFGSP) regroupe la Paces ainsi que les deuxièmes et troisièmes années des études pharmaceutiques. Son programme est défini sur le plan national [4]. II comprend un tronc commun visant à fournir les acquis indispensables pour tout futur pharmacien, indépendamment de la spécialité qu'il souhaite suivre par la suite. L'étudiant bénéficie d'UE laissés à son libre choix pour lui permettre de découvrir les différentes facettes du métier.

TEG1 C'est durant la deuxième année que l'étudiant réalise son premier stage au sein d'une officine. Ce stage, d'observation ou d'initiation, a pour objectif de lui faire découvrir le milieu officinal et les relations que peut entretenir le pharmacien avec les autres professionnels de santé. D'une durée réglementée de six semaines - mais pouvant être réalisé en deux fois dans certaines facultés -, il s'effectue chez un pharmacien agréé maître de stage [5].

TEG1 En troisième année, les étudiants réalisent un nouveau stage en officine, d'une à deux semaines, qui a un tout autre objectif. Il s'agit d'un stage d'application durant lequel ils mettent en application une thématique précise du programme. Ils peuvent alors concrétiser les acquis théoriques appris sur les bancs de la faculté.

T1 Le diplôme de formation appliquée en sciences pharmaceutiques

TEG1 Le diplôme de formation appliquée en sciences pharmaceutiques (DFASP) comprend la quatrième et la cinquième année des études de pharmacie [6]. II permet à l'étudiant de se spécialiser progressivement dans les disciplines de son choix et de parfaire son projet professionnel.

TEG1 Le premier semestre et une partie du second semestre de la quatrième année font partie du tronc commun. Les étudiants y terminent l'apprentissage de l'enseignement théorique et une nouvelle mise en application est prévue au travers d'un nouveau stage, lui aussi d'une durée d'une à deux semaines.

TEG1 Les étudiants ont le choix entre différents parcours à la fin du premier semestre de la quatrième année : officine, internat, industrie/recherche (le parcours recherche est parfois individualisé). C'est donc au second semestre que débute la spécialisation. Les parcours officine et industrie/recherche oriente vers un troisième cycle court alors que la filière internat débouche sur un troisième cycle long. Les étudiants qui s'y destinent doivent passer un concours durant leur cinquième année.

TEG1 La cinquième année se concentre sur les enseignements spécialisés selon le parcours choisi. L'étudiant doit réaliser un stage d'une durée de six mois équivalent temps plein au sein d'un centre hospitalier (dans différents services et possiblement à l'étranger) pour découvrir ce milieu.

\section{T1 Le troisième cycle}


TEG1 Le troisième cycle court officine, qui accueille des étudiants se destinant à l'exercice officinal, comporte une sixième et dernière année. Les cours prévoient des mises en situation qui permettent d'appliquer les connaissances théoriques accumulées les années précédentes. D'une période de trois à quatre mois, ils précèdent un stage professionnel d'une durée de six mois, réalisé dans une (ou deux) officine(s) de ville.

C'est durant cet ultime stage que l'étudiant se confronte à son futur exercice professionnel. Différentes missions lui sont confiées pour qu'il prenne progressivement conscience de la responsabilité à laquelle il fera face une fois diplômé.

La finalisation d'une thèse d'exercice est indispensable pour obtenir le diplôme d'État de docteur en pharmacie.

TEG1 Le troisième cycle court industrie prévoit un stage dans une entreprise pharmaceutique. Les cours spécialisés qu'ils ont pu suivre au cours d'un master permettent, là encore, aux étudiants de se confronter à leur futur métier et de passer à la pratique. En plus d'une deuxième année de master à orientation professionnelle, ils ont la possibilité d'intégrer un master à orientation recherche (en vue de la préparation d'un doctorat d'université), une école d'ingénieurs ou de commerce.

Tout comme les officinaux, ils doivent soutenir leur thèse d'exercice - et ce, dans les deux ans qui suivent la validation de leur sixième d'année de pharmacie - pour obtenir le diplôme d'État de docteur en pharmacie.

TEG1 Le troisième cycle long internat s'ouvre aux étudiants classés en rang utile au concours national de l'internat. En fonction de leur classement, ils peuvent choisir parmi trois spécialités : pharmacie hospitalière (PH), biologie médicale (BM) et innovation pharmacie et recherche (IPR).

Durant quatre années, les étudiants acquièrent de nouvelles compétences sur différents terrains de stage. Plus les semestres progressent et plus ils gagnent en autonomie et responsabilité. Ils ont également la possibilité de suivre une année-recherche.

À l'issue de ce cycle, l'étudiant soutient un mémoire qui peut tenir lieu de thèse d'exercice, lui permettant d'obtenir son diplôme d'État de docteur en Pharmacie.

\section{Points à retenir}

- Les études pharmaceutiques sont organisées en trois niveaux : diplôme de formation générale en sciences pharmaceutiques, diplôme de formation approfondie en sciences pharmaceutiques et diplôme d'État de docteur en pharmacie.

- Durant la cinquième année dite "hospitalo-universitaire", l'étudiant en pharmacie occupe un poste d'externe au sein d'un centre hospitalier universitaire et découvre le fonctionnement des services hospitaliers.

- Le cycle court officinal comporte un stage obligatoire de six mois de pratique professionnelle. 
- Le certificat de synthèse pharmaceutique (CSP) doit être obtenu pour valider le second cycle des études pharmaceutiques.

- Depuis la rentrée 2018, le CSP a été uniformisé dans toutes les facultés de France, prenant la forme d'un oral.

\section{T1 Le certificat de synthèse pharmaceutique}

TEG1 Une nouvelle épreuve a fait son apparition depuis la dernière réforme des études de pharmacie de 2010 : le certificat de synthèse pharmaceutique (CSP), indispensable pour valider le second cycle.

TEG1 Cette épreuve est présentée comme un garde-fou permettant de s'assurer que tout étudiant a acquis les compétences nécessaires à l'obtention de son diplôme. Elle se déroule en quatrième année (avec des sessions de rattrapages en quatrième et/ou cinquième année si besoin). Un jury pluridisciplinaire évalue l'étudiant sur sa capacité à synthétiser les connaissances acquises au cours du tronc commun du cursus. Le format a été uniformisé sur l'ensemble du territoire sous forme d'oral depuis la rentrée 2018.

\section{T1 Conclusion}

Les études pharmaceutiques sont d'une grande richesse et permettent à tout étudiant de trouver sa voie. Les filières officine et industrie ainsi que l'internat donnent accès à un panel de métiers conséquent. L'acquisition des doubles compétences combinée avec un diplôme d'ingénieur ou d'école de commerce se développe de plus en plus. Aujourd'hui, le pharmacien doit, pour se démarquer, se former pour porter les nouvelles missions qui lui sont octroyées.

\section{Références}

[1] Arrêté du 21 décembre 2015 fixant le nombre d'étudiants de première année commune aux études de santé autorisés à poursuivre leurs études en pharmacie à la suite des épreuves terminales de l'année universitaire 2015-2016 et le nombre d'étudiants pouvant être admis directement en deuxième année de ces études à la rentrée universitaire 2016-2017 en application de l'article 9 du décret $n^{\circ}$ 2014-189 du 20 février 2014 tendant à l'expérimentation de modalités particulières d'admission dans les études médicales, odontologiques, pharmaceutiques et maïeutiques. Journal officiel de la République française du 26 décembre 2015. www.legifrance.gouv.fr/eli/arrete/2015/12/21/AFSH1531334A/jo/texte 
[2] Ministère de l'Enseignement supérieur, de la Recherche et de l'Innovation. Expérimentation des modalités d'admission dans le cadre des études de santé. www.enseignementsup-

recherche.gouv.fr/cid78906/experimentation-des-modalites-d-admission-dans-le-cadre-des-etudesde-sante.html

[3] Tesniere A, Rist S, Riom I. Stratégie de transformation du système de santé. Adapter les formations aux enjeux du système de santé. Rapport final. https://solidaritessante.gouv.fr/IMG/pdf/masante2022_rapport_adaptation_des_formations.pdf

[4] Ministère de l'Enseignement supérieur, de la Recherche et de l'Innovation. Bulletin officiel $n^{\circ} 17$ du 28 avril 2011. www.enseignementsup-recherche.gouv.fr/pid20536/bulletin-

officiel.html?cid_bo=55866

[5] Collège des pharmaciens conseillers et maîtres de stage. http://cpcms.fr/maitres-de-stage/

[6] Ministère de l'Enseignement supérieur, de la Recherche et de l'Innovation. Bulletin Officiel $n^{\circ} 20$ du 16 mai 2013. www.enseignementsup-recherche.gouv.fr/pid20536/bulletin-

officiel.html?cid_bo $=71555 \&$ cbo $=1$

Déclaration de liens d'intérêts

Les auteurs déclarent ne pas avoir de liens d'intérêts.

Figure

\section{Ade_fig1}

Figure 1. Schéma général des études pharmaceutiques en France en 2019.

(C) É. Adenot

Sur 2 colonnes en haut de deuxième page 


\title{
Première année commune des études de santé
}

Professions médicales (pharmacie, médecine, odontologie, maïeutique) +/- professions paramédicales (kinésithérapie, ergothérapie...) selon les facultés

\section{CONCOURS}

\author{
Deuxième année de pharmacie \\ 6 semaines de stage d'initiation en officine
}

Troisième année de pharmacie

1 à 2 semaines de stage d'application en officine

\section{Quatrième année de pharmacie}

1 à 2 semaines de stage d'application en officine

Début de la filialisation au second semestre

\section{Cinquième année de pharmacie}

4 à 6 mois équivalent temps plein de stage hospitalier
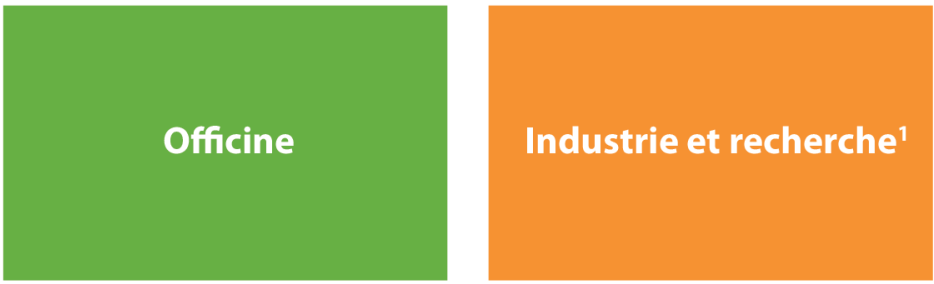

Préparation internat

\section{DES - Sixième année de pharmacie - officine 6 mois de stage en officine}

Thèse d'exercice
DES - Sixième année de pharmacie - industrie

6 à 12 mois de stage

Thèse d'exercice

\section{CONCOURS}

DES - Internat ${ }^{2}$ 4 ans d'internat Stage à l'hôpital, un service différent par semestre

${ }^{1}$ Ces filières sont distinctes dans certaines facultés.

${ }^{2}$ Trois filières possibles : biologie médicale (BM), pharmacie hospitalière (PH), innovation pharmaceutique et recherche (IPR). 